\title{
Eruption of a Large Quiescent Prominence and Associated CME on 2001 January 14
}

\author{
S. L. Ma, Y. C. Jiang, Q. Y. Li, S. Q. Zhao, L. P. Li, and H. D. Chen \\ National Astronomical Observatories/Yunnan Astronomical Observatory, CAS, Kunming \\ 650011, China (email: jyc@ynao.ac.cn)
}

\begin{abstract}
We present observations of a spectacular eruption of a huge quiescent prominence, which was clearly associated with a coronal mass ejection (CME). The CME consisted of a typical three-part structure: a bright loop-like leading edge, a dark cavity and a bright core. The prominence exhibited a very symmetrical loop-like eruption in low corona and matched well with the bright CME core trailing the CME leading edge. By combining the $H_{\alpha}, 17 \mathrm{GHz}$ and EUV observations with white-light coronagraphs observations, the bright CME core was conclusively identified as the erupting cool, dense prominence material.
\end{abstract}

Keywords. Sun: activity, coronal mass ejections (CMEs), prominences

On 2001 January 14, a large quiescent prominence erupted in the north-western solar limb and an associated partial halo-type CME was well observed by the Large Angle and Spectrometric Coronagraphs (LASCO) on the Solar and Heliospheric Observatory (SOHO). In this paper, we investigate this prominence eruption and its relationship with the CME using $H_{\alpha}$ data from Yunnan Astronomical Observatory (YNAO), $17 \mathrm{GHz}$ microwave data from the Nobeyama Radioheliograph, EUV data from the Extremeultraviolet Images Telescope (EIT) on the SOHO, as well as the LASCO white light coronagraphs data.

The prominence activity started at approximately 03:00 UT, and began to ascend at about 04:30 UT. $H_{\alpha}, 17 \mathrm{GHz}$ and EIT $195 \AA$ observations show that this was a very symmetrical loop-like eruption (see Figure 1(a1-a3), (b1-b3) and (c1-c3), respectively) . The whole prominence body lifted up while its two legs stayed anchored (see the inner EIT $304 \AA$ image in Figure $1(d)$ ). According to the EIT $195 \AA$ observations, the height, speed and acceleration for the prominence are measured and plotted in Figure 2. It is noted that no GOES flare was recorded to associate with the eruption (see Figure $2(b)$ ).

The CME with a central position angle of $356^{\circ}$ and a width of $134^{\circ}$ was first seen in LASCO C2 images at 06:54 UT as a bright loop. The CME consisted of a typical three-part structure: a bright loop-like leading front, a dark cavity and a bright core. There were two bright legs connecting the bright core back beneath the occulting disk. Figure $1(d)$ shows that the bright CME legs matched well with the legs of the erupting prominence (indicated by the arrows), definitely indicating that the CME core was the erupting cool, dense prominence material in the outer corona. This is consistent with the results of Dere, et al. (1997) and Plunkett, et al. (2000). The CME front heights measured by Seiji Yashiro are shown in Figure 2(a), and the average velocity and acceleration from the first and second-order polynomial fits are $945 \mathrm{~km} \mathrm{~s} s^{-1}$ and $24.7 \mathrm{~m} \mathrm{~s}^{-2}$, respectively. Therefore, this was a fast CME although it was correlated with the prominence eruption.

\section{Acknowledgements}

We thank the Nobeyama Radioheliograph group, the EIT and LASCO teams for data support. The work is supported by the NSFC under grant 10173023 . 


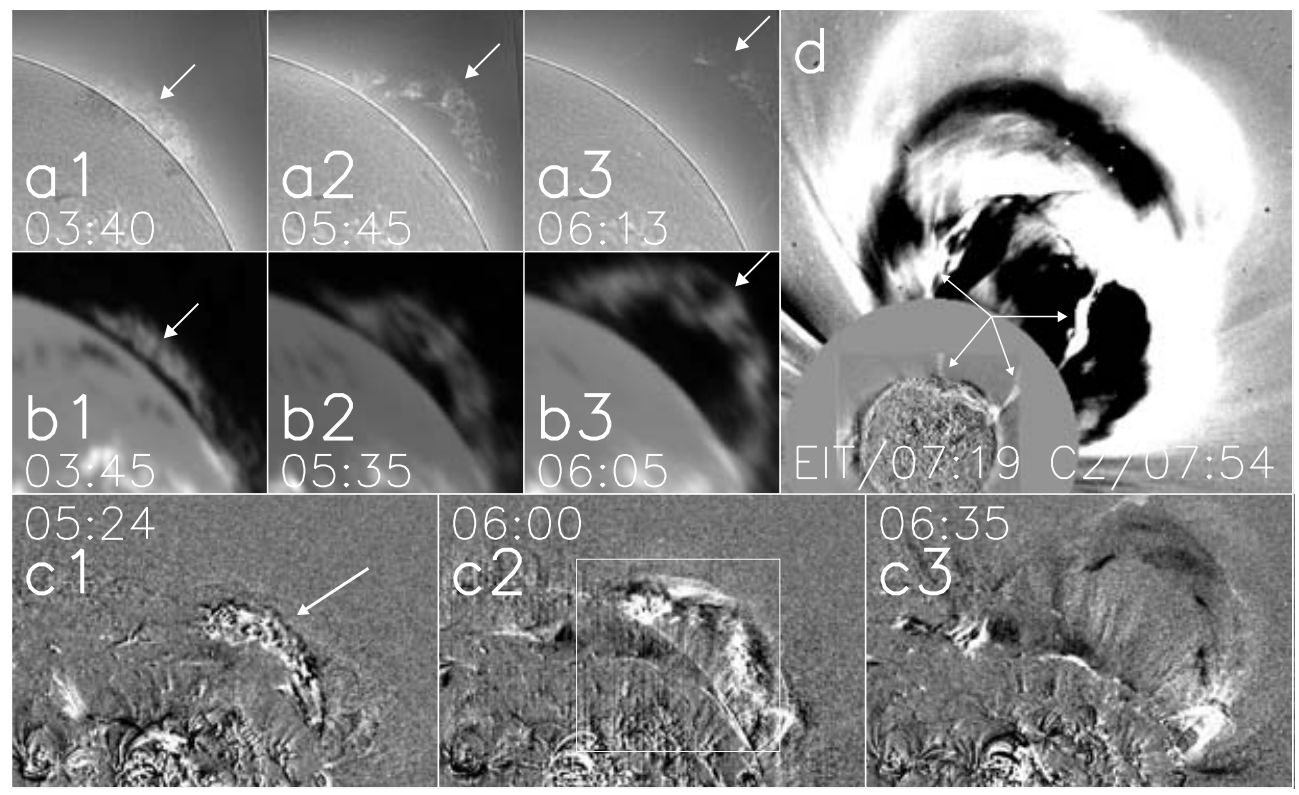

Figure 1. YNAO $H_{\alpha}$ (a1-a3), Nobeyama $17 \mathrm{GHz}$ microwave (b1-b3) and EIT $195 \AA$ difference images (c1-c3) showing the prominence eruption (see the arrows); the white box in panel c2 indicates the field of view of $H_{\alpha}$ and $17 \mathrm{GHz}$ images. The panel $\mathrm{d}$ is a composite image of inner $304 \AA$ with outer LASCO C2 difference images. The arrows indicate the legs of the erupting prominence and the CME core.

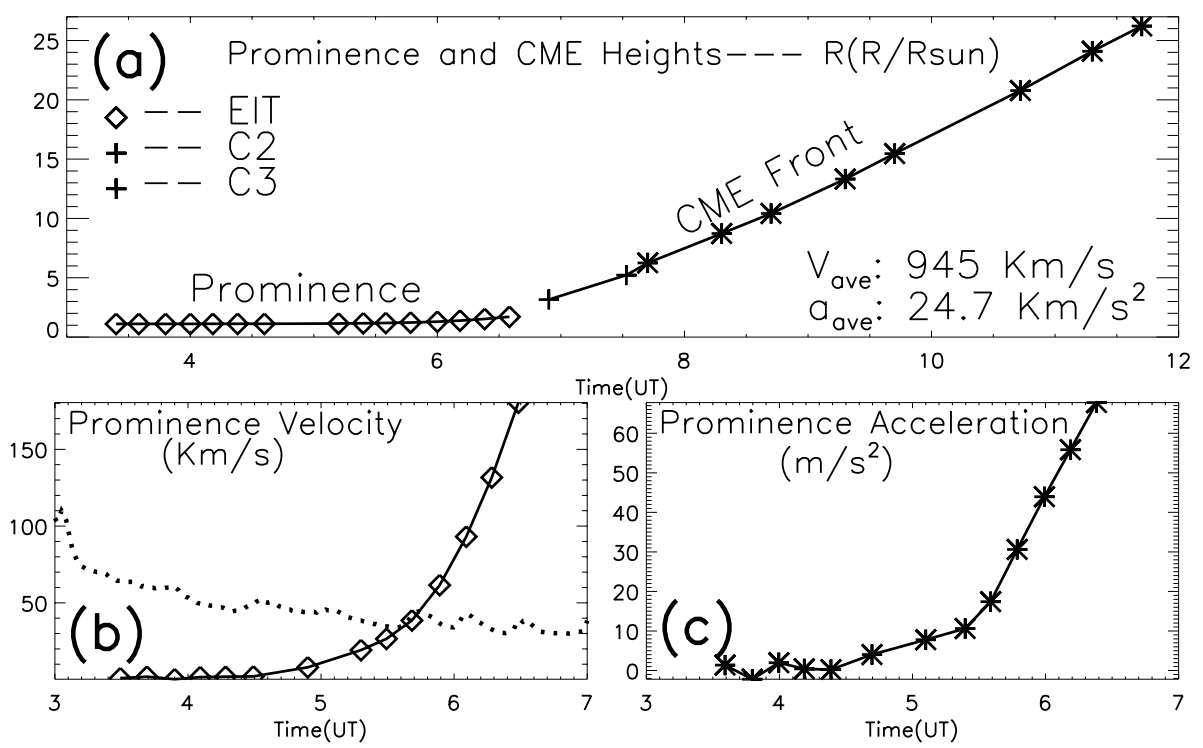

Figure 2. Plots showing heights for the prominence and CME (a), speed (b) and acceleration (c) for the prominence. The dashed line in panel b showing time profiles of GOES-8 soft X-ray.

\section{References}

Dere, K. P., et al. 1997, Solar Phys. 175, 601

Plunkett, S. P., et al. 2000, Solar Phys. 194, 371 